\title{
Bridging cultural sociology and cognitive psychology in three contemporary research programmes
}

\begin{abstract}
Michèle Lamont ${ }^{1,2 \star}$, Laura Adler ${ }^{3}$, Bo Yun Park ${ }^{3}$ and Xin Xiang ${ }^{4}$
Three prominent research programmes in cognitive psychology would benefit from a stronger engagement with the cultural context of cognition: studies of poverty focused on scarcity and cognitive bandwidth, studies of dual-process morality and studies of biases using the implicit association test. We address some limitations of these programmes and suggest research strategies for moving beyond an exclusive focus on cognition. Research on poverty using the cognitive bandwidth approach would benefit from considering the cultural schemas that influence how people perceive and prioritize needs. Dual-process morality researchers could explain variation by analysing cultural repertoires that structure moral choices. Research using the implicit association test can better explain implicit attitudes by addressing the variability in cultural schemas that undergird biases. We identify how these research programmes can deepen the causal understanding of human attitudes and behaviours by addressing the interaction between internal cognition and supra-individual cultural repertoires.
\end{abstract}

I $\mathrm{n}$ recent years, social scientists have increasingly turned to individual cognitive processes to explain social phenomena. Three prominent manifestations of this trend are the study of poverty focused on scarcity and cognitive bandwidth (CB); the study of dual-process morality (DPM), which describes how moral judgement differs between automatic and reflective thought; and the study of people's attitudes through implicit association tests (IATs). Each yields important insights into complex social processes. $\mathrm{CB}$ provides a cognitive explanation for why low-income people make decisions that extend their poverty. DPM uncovers the psychological processes that produce puzzling patterns of moral judgements. IAT reveals otherwise unobservable biases that can lead to discrimination.

As each line of work has gained extraordinary prominence in academic and policy discussions, they deserve close examination. Building on behavioural economics, the CB model has led policymakers to mobilize psychological 'nudges' to achieve specific goals, such as helping the poor save money. DPM has contributed to the proliferation of programmes in 'mind, brain and behaviour' and business ethics across US universities. Over 14 million people have taken IATs and the method has been adopted by employers, such as Google and the Chicago Police Department, as a tool for promoting inclusion by attuning participants to their own biases. While much differentiates these three lines of work, which range from an integrated research programme to a wide-ranging body of research mobilizing a common tool, they share a relative prominence, a focus on cognition and an aspiration to explain important social phenomena.

These influential approaches also have a common limitation: each explains judgements and behaviours by cognition alone, skirting the cultural background that makes some cognitive referents more available or salient than others ${ }^{1-3}$. In focusing on universal cognitive processes, they present culturally specific worldviews as universal or natural. To explain meaningful variations in judgement and behaviour across groups, these approaches must consider the group-level cultural repertoires that undergird cognition. Specifically, we propose that universal cognitive processes are shaped by the specific cultural repertoires provided by the social environment, which vary between cross-cutting social groups. Stronger causal explanations can thus be produced by bridging research on cognition with cultural sociology.

Building on Emile Durkheim's work on classification systems, cultural sociologists have emphasized 'cultural repertoires', defined as the available schemas, frames, narratives, scripts and boundaries that actors draw on in social situations (for definitions, see ref. ${ }^{4}$ ). This influential model of culture diverges from older models that implied cultural homogeneity within groups, such as those bound by common national membership ${ }^{5}$. In a classic paper, Swidler emphasized the pragmatic use of meanings and symbols in everyday life, arguing that "culture influences action not by providing the ultimate values toward which action is oriented but by shaping a repertoire or 'tool-kit' of habits, skills, and styles"'. The concept of cultural repertoires thus emphasizes the heterogeneity of cultural tools; the varying availability of these tools between cross-cutting groups such as those defined by nationhood, gender, ethnicity, age or class; and the non-deterministic character of cultural toolkits, which enable and constrain rather than prescribing patterns of thought and behaviour.

Subsequent research has examined why actors use some cultural tools instead of others. Social environments provide people with specific meanings: the social world is suffused with symbolic systems - ranging from national myths, such as the American dream, to scripts about gender roles - which are diffused by intermediaries ranging from religious groups and the media to scientific and legal experts, making specific cultural referents more or less salient to members of different social groups. For instance, Lamont and

'Department of Sociology, Department of African and African American Studies, and Weatherhead Center for International Affairs, Harvard University, Cambridge, MA, USA. ${ }^{2}$ Canadian Institute for Advanced Research, Toronto, ON, Canada. ${ }^{3}$ Department of Sociology, Harvard University, Cambridge, MA, USA. ${ }^{4}$ Harvard Graduate School of Education, Harvard University, Cambridge, MA, USA. Laura Adler, Bo Yun Park and Xin Xiang contributed equally to this work. *e-mail: mlamont@wjh.harvard.edu 
Table 1 | Characteristics of the three research programmes and propositions for future research

\begin{tabular}{|c|c|c|c|}
\hline & Cognitive bandwidth research on poverty & Dual-process morality research & Implicit association test research \\
\hline Proposition & $\begin{array}{l}\text { The behaviours of the poor are shaped by } \\
\text { conditions of scarcity that tax cognitive } \\
\text { bandwidth and lead to non-optimal } \\
\text { decisions. }\end{array}$ & $\begin{array}{l}\text { We have two modes of cognition, } \\
\text { automatic and reflective, that are typically } \\
\text { associated with distinct types of moral } \\
\text { judgement, deontological and utilitarian. }\end{array}$ & $\begin{array}{l}\text { People have implicit biases that can lead } \\
\text { to discriminatory behaviour. The IAT } \\
\text { unveils the implicit biases that cannot be } \\
\text { captured by self-reporting. }\end{array}$ \\
\hline Key texts & Ref. ${ }^{18}$ & Refs 35,79 & Refs 55,64 \\
\hline $\begin{array}{l}\text { How the } \\
\text { approach } \\
\text { naturalizes } \\
\text { cultural } \\
\text { repertoires }\end{array}$ & $\begin{array}{l}\text { Without investigating variation in } \\
\text { perceptions of scarcity, CB presumes } \\
\text { a natural or inevitable prioritization of } \\
\text { multiple scarce resources. }\end{array}$ & $\begin{array}{l}\text { By associating automatic cognition with } \\
\text { deontological judgement and reflection } \\
\text { with utilitarian judgment, DPM presumes } \\
\text { that these Western moral schemas are } \\
\text { universal. }\end{array}$ & $\begin{array}{l}\text { Without accounting for the available } \\
\text { cultural schemas, IAT cannot disentangle } \\
\text { different possible meanings associated } \\
\text { with differential response times. }\end{array}$ \\
\hline $\begin{array}{l}\text { Consequences } \\
\text { of naturalizing } \\
\text { cultural } \\
\text { repertoires }\end{array}$ & $\begin{array}{l}\text { Without understanding the cultural } \\
\text { repertoires that lead to diverse perception } \\
\text { and prioritization of scarce resources, } \\
\text { CB research lacks a causal model for } \\
\text { understanding the behaviour of the poor. }\end{array}$ & $\begin{array}{l}\text { Without considering the diversity of } \\
\text { cultural repertoires, DPM research cannot } \\
\text { explain observed patterns of variation } \\
\text { across groups or provide reliable guidance } \\
\text { for resolving moral conflicts or reducing } \\
\text { ethical transgressions. }\end{array}$ & $\begin{array}{l}\text { Without considering the cultural } \\
\text { repertoires that shape ideas about social } \\
\text { groups, IAT research cannot determine } \\
\text { whether measurable biases are evidence } \\
\text { of relative salience or substantive } \\
\text { (good/bad) evaluation. }\end{array}$ \\
\hline $\begin{array}{l}\text { Propositions } \\
\text { for research } \\
\text { integrating } \\
\text { cultural } \\
\text { repertoires }\end{array}$ & $\begin{array}{l}\text { CB research can incorporate existing } \\
\text { research on cultural repertoires to better } \\
\text { interpret results and include questions } \\
\text { about the meaning of multiple scarce } \\
\text { resources in research designs. Cultural } \\
\text { sociologists can extend CB insights by } \\
\text { investigating the meaning and evaluation } \\
\text { of diverse scarce resources. }\end{array}$ & $\begin{array}{l}\text { DPM research can design new moral } \\
\text { dilemmas based on diverse moral } \\
\text { discourses, and investigate how certain } \\
\text { schema become 'intuitive' or the default of } \\
\text { moral reflection. Cultural sociologists can } \\
\text { elicit and analyse people's explanations of } \\
\text { their responses. }\end{array}$ & $\begin{array}{l}\text { IAT research can incorporate existing } \\
\text { research in cultural sociology to better } \\
\text { understand potential heterogeneity in } \\
\text { results, and include questions about } \\
\text { exposure and schemas of stigmatization } \\
\text { into research designs. Cultural sociologists } \\
\text { can consider findings from cognitive } \\
\text { science to better understand people's } \\
\text { reaction to discriminatory attitudes. }\end{array}$ \\
\hline
\end{tabular}

Thévenot ${ }^{7}$ showed that people in the United States are more likely to make evaluations based on economic criteria, while in France, referents about civic solidarity and aesthetics are more common. At the sub-national level, literature on institutional logics examines variation in the salience of schemas, frames, narratives and boundaries across organizations, classes and occupations (for example, ref. ${ }^{8}$ ). Still others show how cultural tools become available to different groups: young working class men in the United Kingdom learn to perform masculine identity in class-specific ways through interactions at school'; and US women learn to behave in accordance with 'intensive mothering' in response to the diffusion of gendered ideologies via child-rearing books ${ }^{10}$. This literature often draws on interviews, but it does not assume that self-reports reflect behaviour; instead it aims to document the relative salience of these repertoires ${ }^{7}$. Such work shows that differently structured social environments make some cultural referents more readily available to individuals than others. Such cultural meanings, in turn, can shape individual cognition.

This literature complements the path-breaking work of cultural psychologists who have examined the cultural foundation of individual cognition, often using data from other societies to challenge universalist assumptions in mainstream psychology (for example, refs ${ }^{11-14}$ ). While cultural psychologists demonstrate national, regional and class variations (for example, ref. ${ }^{15}$ ), they often assume cultural homogeneity within groups or societies and are less concerned with identifying why some schemas are more readily available than others. Our objective differs from that of cultural psychologists in that we offer new insight into unexplained sources of variation by foregrounding causes of cultural heterogeneity (for example, ref. ${ }^{16}$ ).

Because cultural repertoires include schemas that structure the valuation of scarce resources, widely shared concepts of morality and the stigmatization of groups, research in CB, DPM and IAT would benefit from a deeper engagement with cultural variation. By addressing the cultural repertoires through which groups think, feel and act, these research programmes can enrich the understanding of why the universal cognitive processes posited by CB, DPM and IAT produce particular patterns of judgements and behaviours in specific contexts.

Table 1 summarizes the three approaches, the consequences of narrowly focusing on individual cognition and naturalizing cultural repertoires, and propositions for integrating the study of universal cognition and cultural repertoires. The latter enable the very categories needed to conceptualize the study of poverty, morality and discrimination: in $\mathrm{CB}$, the idea that some resources are particularly valuable; in DPM, the various moral concepts constituting moral worth; and in IAT, the idea of group difference. By ignoring variations in cultural background, these approaches can yield inaccurate predictions about responses to scarcity, lead to misguided prescriptions for moral reasoning, and prevent researchers from distinguishing between evaluation and salience of categories in producing implicit bias.

In the following sections, we turn to the main argument of the three programmes, what we believe they miss and how to build bridges between universal cognition and cultural repertoires. We conclude by offering preliminary proposals for building an analytical bridge with cultural sociology.

\section{The cognitive bandwidth model}

Scholars have long debated why the poor so often remain in poverty. Culture of poverty arguments, which suggest that the poor are inhibited by deviant values or habits ${ }^{17}$, have fallen out of favour ${ }^{4}$. However, social scientists recognize that the poor often make decisions that prolong or deepen their disadvantage. The CB approach provides new insights into cognitive processes underlying these behaviours by examining decision-making in the context of scarcity. The universal cognitive processes it identifies reveal commonalities between the poor and the middle class that help readers understand apparently irrational behaviour. Without investigating the diverse cultural repertoires that structure cognition, however, 
the model is insufficient to explain actual behaviour in specific contexts.

In Scarcity ${ }^{18}$ and related articles (for example, refs ${ }^{19,20}$ ), economist Sendhil Mullainathan and psychologist Eldar Shafir propose that when people feel they have insufficient time, money or food, they focus exclusively on managing the scarce resource. This heightened focus, or 'tunnelling', has benefits: by ignoring irrelevant issues, we make better use of cognitive capacities to solve the focal problem, yielding a 'focus dividend'. People with scarce resources are more economically rational with regard to the specific scarce resource than those with abundant resources ${ }^{21}$.

This tunnelling, however, reduces the amount of cognitive 'bandwidth' available for other concerns, creating new problems. For the poor, the pressing need to focus on short-term economic requirements inhibits long-term planning, leading to future scarcity; tunnelling thus becomes not only an effect but also a cause of poverty. For the businessman with scarce time to meet work deadlines, tunnelling will lead him to neglect his family: 'projects must be finished now; the children will be there tomorrow'18.

The CB model helps explain routine non-optimal decisionmaking, drawing parallels between the cash-strapped poor and the time-strapped middle class to highlight the universal effects of scarcity on cognition. But the model is limited by the assumption that there is universal agreement about which resources are most essential. In fact, people often experience multiple simultaneous forms of scarcity. This is particularly true among the poor, for whom health problems, economic insecurity, housing instability and violence frequently coincide ${ }^{22}$.

In presuming that economic scarcity is always the most salient to the poor, $\mathrm{CB}$ research risks labelling behaviours that enable survival in specific contexts as 'non-optimal'. In both rural India and the United States, poor people spend scarce money on social functions such as celebrations ${ }^{23}$ or funerals ${ }^{24}$, because the social cost of failing to contribute outweighs competing economic considerations. Similarly, in the hypothetical but intuitive example of the busy working father, it might seem obvious that work demands are more urgent than family demands. But this interpretation relies on a particular set of cultural interpretations: specific scripts of fatherhood, frames that distinguish 'work life' from 'family life' and narratives linking self-worth to economic success.

Research shows, however, that cultural repertoires vary across social groups, such that which problem takes priority during tunnelling is not obvious. Our intuitive expectation for the businessman may be different if the working parent were a woman. Even among men, Waller ${ }^{25}$ has demonstrated that non-resident, lowincome fathers frequently repurpose the concept of 'the provider' to emphasize emotional presence over material resources, suggesting that men prioritize family time in certain circumstances. While the cognitive process of 'tunnelling' may take place under conditions of scarcity, without considering the role of cultural repertoires in structuring the perception of scarcity and the evaluation of competing scarce resources, we cannot develop a causal understanding of decision-making.

By incorporating cultural repertoires, CB research can develop a richer model of how the poor or the middle class perceive, evaluate and respond to situations of scarcity. Hierarchies of concern are far from universal; culturally available frames influence which of several competing needs takes priority to become the object of 'tunnelling. As CB influences the design of policy interventions directed at poor communities (for example, ref. ${ }^{26}$ ), we risk implementing anti-poverty interventions detrimental to material, social and cultural well-being by not considering what specific groups value and why.

Bringing culture in. The CB approach can develop more accurate models of decision-making by addressing cultural variations. We adopt the view that the poor do not 'have' a culture that explains their poverty; rather people living in poverty 'use and create symbols' that give meaning to their context and inform strategies of action $^{4}$. Our proposal, therefore, is that studies of decision-making under scarcity consider cultural repertoires available in a given social context to enrich the understanding of how people perceive and evaluate their needs. Where the CB model makes assumptions about the perception and prioritization of scarce resources, it will overlook ways in which apparently non-optimal behaviour, in fact, responds skilfully to the specific priorities of individuals and groups.

Rosen ${ }^{27}$ studied why residents of poor neighbourhoods decide to move, although moves - typically costly - are mostly 'horizontal', between neighbourhoods that are similar in terms of safety and poverty. In unsafe neighbourhoods, residents construct narratives of safety to 'emphasize their own ability to cope ${ }^{27}$. When they can maintain these narratives, they typically remain in place - even in the face of real threats. When events disrupt their narrative frameworks, they often move - even if moving provides no actual improvement in safety. This work demonstrated that cultural repertoires shape prioritization: where we might expect poor residents to prioritize urgent economic demands, respondents take on the cost of moving because narratives of safety are more pressing than economic concerns. Narratives thus determine which resource becomes the focus of tunnelling. That 'tunnelling' on safety does not yield more 'rational' outcomes with respect to this scarce resource raises the question of whether the CB model's 'focus dividend' applies equally to non-economic resources.

In the world of professionals, Blair-Loy's study of women finance executives ${ }^{28}$ showed that repertoires inform prioritization of multiple scarce resources for women balancing work and family. She identified inductively a 'work devotion schema', traditionally associated with men, which demands dedication of time and loyalty to the career; and a 'family devotion schema', which demands that women prioritize family and home above all. Such schemas change over time: older cohorts are more likely to accept the irreconcilability of these demands and abstain from childrearing, while younger cohorts rearticulate the family devotion schema to enable the coexistence of commitments. Blair-Loy's work highlights the gendered cultural repertoires underlying the tendency posited by Mullainathan and Shafir for a hypothetical father to 'tunnel' on work instead of family. It also demonstrates the evolution of repertoires in response to changing social conditions.

These studies suggest that causal understanding of decisionmaking can be improved by considering empirical findings from cultural sociology in field and experimental studies of decisionmaking under scarcity. Drawing on the literature documenting cultural repertoires, researchers can identify additional explanations for existing findings. For instance, economic sociologist Zelizer and colleagues ${ }^{29}$ highlighted the multiple meanings of money to argue that behavioural economists should regard the significance of economic maximization as highly variable. Future research should clarify causal mechanisms by gathering data about the existence and evaluation of multiple forms of scarcity. For instance, in field studies, scholars can specify the effect of scarcity on cognition using questions that variably highlight economic, familial, safety, moral or health-related resources.

Cultural sociologists, for their part, can take inspiration from $\mathrm{CB}$ research to investigate the meanings attached to diverse scarce resources and how they structure variations in behaviour. Daniel ${ }^{30}$, for instance, showed that low-income parents hesitate to introduce new foods to their children because the repeated trials required to acclimate children to new tastes are costly. Where low-income parents see rejected food as an economic loss, higher-income respondents frame this as an experiential gain. This work demonstrates the opportunities for cultural sociologists to improve their 
understanding of class cultures by creating analytical bridges with cognitive psychology.

\section{Dual-process morality}

The question of whether intuition or reasoning drives moral decisions is an old puzzle in psychology ${ }^{31-33}$. Over the past decade, a group of moral psychologists answered this question with their research programme on DPM, which suggests that moral judgements and decisions are shaped by the interaction between two distinct cognitive processes: one fast, unconscious and automatic; the other slow, reflective and effortful ${ }^{34,35}$.

Much of this work analyses responses to hypothetical scenarios such as the classic 'trolley problem': a runaway trolley is about to kill five people; the only way to save them is to pull a switch directing the trolley to another track where one person, instead of the five, will be killed. In a variant called the 'footbridge' version, the respondent contemplates pushing another person wearing a heavy backpack off a footbridge in front of the trolley to stop it from killing the five. Research, mostly with US and European participants, shows that while the majority of people approve of pulling the switch, only a minority considers pushing the backpacker morally acceptable ${ }^{36}$.

DPM researchers argue that the automatic process is driven by deep-rooted intuitions that associate violent acts - such as pushing someone off a bridge - with strong negative affect, thereby producing judgements aligned with deontological principles designating the act of killing as fundamentally wrong regardless of its consequences. In contrast, they argue, reflective cognition performs rational analysis of consequences and tends to produce utilitarian judgements, according to which it is morally permissible to sacrifice one to save many (for example, refs ${ }^{37-39}$ ).

Judgements and decisions depend on the relative strength of the output of the two systems $\mathrm{s}^{35,39}$, which can be changed with selective interventions in experimental settings. A heavy cognitive load (produced by asking a subject to engage in a distracting memorization task, for instance) consumes cognitive resources and makes people more likely to uphold their deontological principles ${ }^{40}$. In contrast, brain-lesion patients with dampened emotions exhibit exceptionally high levels of utilitarian judgements ${ }^{35}$. Business ethics programmes thus draw on DPM, aiming to reduce ethical transgressions by encouraging people to shift between automatic and reflective thinking (see, for example, the 'Ethics Unwrapped' programme at the University of Texas at Austin and the 'behavioural ethics' symposium held at Harvard Business School).

DPM research increasingly predefines specific schemas, frames, discourses and categorization systems undergirding moral judgements as natural or universal. This is apparent in the taken-forgranted association of 'utilitarian judgement' with reflective cognition, and of 'deontological judgement' with automatic cognition. Moreover, the negative affective responses produced by automatic processes in reaction to harming others (such as pushing another person off a bridge) are described as the natural result of evolution that promoted the species' chances of survival, while the 'utilitarian' tendency to tolerate harm to one person to save five others is naturalized as universal human rationality ${ }^{36,41,42}$. Yet, no empirical evidence of such associations is provided.

While the dual-process structure of moral cognition may well be universal, the specific kinds of judgements that automatic and reflective processes produce - and when they are at odds with one another - are shaped by cultural repertoires about morality. Naturalizing particular moral discourses limits DPM researchers' ability to describe and explain variations in moral judgements across contexts. For example, Xiang ${ }^{43}$ found that Tibetan Buddhist monks and lay Tibetans overwhelmingly give 'utilitarian responses' even in the more emotionally disturbing footbridge version. DPM cannot convincingly account for such variations because it does not consider the life worlds of diverse respondents and the cultural context of moral choices. Without analysing the latter, it may be ineffectual and even risky to promote shifting the balance of moral evaluation between automatic and reflective cognition as a means to reduce moral conflicts or transgressions. One need not look beyond moral psychology and behavioural ethics to realize that moral intuitions are not necessarily deontological and moral reflection is not necessarily utilitarian, particularly outside the pool of WEIRD (Western educated industrialized rich democratic) participants ${ }^{44,45}$.

Bringing culture in. To understand the socially and culturally contingent nature of findings from DPM research, we must attend to cultural repertoires about morality - the moral schemas, categorizations and discourses undergirding our moral judgements and behaviours, or what Abend ${ }^{2}$ refers to as 'moral background', which acts as the very foundation enabling assertions of goodness. The intractability of the trolley dilemma is based on particular cultural schemas: the idea of an objective 'greater good' - measured in the number of lives saved - which serves as the basis of evaluating the moral acceptability of an action, and the categorization of the (imagined) action of pushing a person off a footbridge (even if unconsciously) as 'violence', 'killing' or 'inflicting severe harm'. While these particular schemas are commonplace in many Western societies, they are not always and equally present, nor are they the only ones available.

Documenting the empirical cultural repertoires that prevail in the context of moral decision-making could provide more compelling explanations for how universal cognitive processes produce different patterns of moral judgement across contexts. Tibetan respondents may react differently to the trolley dilemma than US respondents because they draw on different repertoires. Indeed, Xiang ${ }^{43}$ showed that US respondents talk about 'rights' or 'better results' when accounting for their preference - consistent with the utilitarian-deontology dichotomy - while Tibetan respondents cite stories from Buddhist sutras to justify using 'compassionate intention' to evaluate the moral goodness of an act, referring to moral categories distinctive to Tibetan Buddhism such as 'karma', 'sacrifice' and 'the heart of a Buddha/bodhicitta' (this contrast between Western and Tibetan Buddhist moral discourses is also discussed by Flanagan ${ }^{46}$ ). Some of the monks argued that pushing the backpacker off the bridge to save others is regarded as morally permissible, or even honourable, if this is done purely out of compassion for the would-be victims without any selfish intentions (such as personal animus towards the backpacker or personal affection for any of the would-be victims lying on the track). This case provides evidence of diversity in moral schemas and logics that DPM researchers miss, including schemas antithetical to the utilitarian and deontological principles taken for granted by this line of research. Moreover, an understanding of cultural repertoires is thus crucial to developing the prescriptive implications of DPM research for resolving moral conflicts or reducing ethical transgressions. Before asking people to shift from automatic to reflective mode or vice versa, researchers should consider the schemas and logics that structure moral intuitions and moral reasoning in a particular social group.

Studies of diverse moral judgements and behaviours suggest that scholars can enrich DPM research by integrating empirical findings from cultural sociology into future studies. To test the universality of the dominant schema, DPM scholars can record explanations of responses to hypothetical scenarios and analyse the alignment with the utilitarian-deontology dichotomy. Rather than relying on moral dilemmas from Western moral philosophers, such as the trolley problem, they could design a variety of moral dilemmas highlighting alternative moral principles documented or analysed by sociologists (for example, varying frames about moral worth among French and US citizens described by Lamont ${ }^{47,48}$ and also by Boltanski and Thévenot ${ }^{49}$, and the different moral narratives of political conservatives and liberals examined by McAdams et al. ${ }^{50}$ ), anthropologists 
(for example, different assumptions about other actors' moral intentions discussed by Keane ${ }^{51}$ ), philosophers (for example, the contrast between Confucian, Buddhist and Western moral discourses presented by Flanagan ${ }^{46}$ ) and other moral psychologists (for example, the diversity of moral intuitions described in Haidt's social intuitionist model $^{33}$ ).

Cultural sociologists can also contribute by collecting data on how individuals interpret situations - data often dismissed as post-hoc rationalizations by cognitive psychologists ${ }^{52}$ - to develop inductive insights into the cultural tools used by diverse respondents in moral situations. By bridging cognitive psychology and cultural analysis, scholars can foreground the often taken-for-granted cultural repertoires that structure automatic or reflective cognition, thus enabling DPM to build more robust causal connections between cognitive processes and patterns of moral decisions in the real world. Beyond the individual moral judgement, scholars can furthermore investigate how certain moral schemas, logics or scripts become 'intuitive' or default formulas for moral judgement, pointing the way to strategies for resolving moral conflicts or reducing ethical transgression.

\section{The implicit association test}

First introduced by Greenwald and colleagues in 1998, the IAT has emerged as one of the most widely used measures of implicit attitudes. Because of its capacity to detect biases that are difficult to capture through self-reporting, it quickly diffused beyond psychology $^{53,54}$ and is now widely used in diversity training in both the public and private sectors.

The IAT analyses a subject's automatic evaluations of an object or a person based on the idea of 'differential association' ${ }^{55}$, which is assessed by measuring the time that respondents take to associate the visual representation of items (for example, faces representing race or gender) with evaluations of certain kinds (for example, good or bad). The matching times between items and evaluative categories predict the existence of an implicit bias or preference vis-à-vis the classified item: the longer the matching time, the more likely it is that there is a bias towards the item.

The method has been subjected to scrutiny, primarily on three grounds: the validity of the test, the degree to which it predicts discriminatory behaviour, and the receptivity of implicit biases to different types of interventions. While the IAT is the object of considerable controversy among organizational psychologists ${ }^{56}$ and social psychologists ${ }^{57}$, as well as in the popular press ${ }^{58}$, the consensus is that it outperforms other indirect measures of biases in terms of internal validity ${ }^{5,59}$. In contrast, the debate over the predictive validity of the IAT is not resolved ${ }^{57}$ : some argue that IAT results are correlated with discriminatory behaviour ${ }^{60,61}$; others claim that the IAT has limited capacity to predict discrimination ${ }^{56,57}$. Finally, scholars disagree about whether implicit biases are immune to outside stimulus ${ }^{62}$.

Regardless of where they stand on these debates, IAT researchers collectively focus on internal cognitive processes to explain attitudes and judgements, rather than addressing the role of cultural repertoires in structuring bias. They implicitly frame cognition as static - concerning 'warm' feelings towards groups, for instance - precluding consideration of changes in bias over time or across context. For example, they do not take into account the national variations in the stigmatization of minority groups ${ }^{16}$. Their static understanding of cultural scripts is also reflected in their methods of 'counterbalancing': IAT researchers attempt to limit the impact of what they regard to be a 'universal norm against darker skin' by simply using grey pixels to represent African Americans.

Though some acknowledge that the outside environment shapes biases $^{63}$, IAT researchers typically operationalize context in rather limited ways. For instance, the test available on the Project Implicit website (the most prominent IAT website) incorporates only a few factors, such as the respondent's zip code ${ }^{64}$. The online test asks respondents to provide information about socio-demographic characteristics (for example, date of birth, race, ethnicity, gender and political identity), but does not collect data on contextual factors that are likely to bear on the nature of biases, such as the frequency of interaction with groups presented in the test (for example, the number of minorities living in one's neighbourhood) or the extent to which an ethno-racial group is stigmatized in the subject's environment. Not factoring in important information about available cultural repertoires limits IAT researchers' capacity to understand the social meaning of implicit bias.

Bringing culture in. Neglecting the cultural repertoires that undergird cognitive processes has important consequences. One of them is that IAT research cannot specify the meaning of differential associations. Most importantly, researchers cannot determine whether the speed of association between image and word categories is due to a positive and negative evaluation, or to relative exposure and familiarity. For instance, faster association between 'good/bad' and Barack Obama - compared with Ronald Reagan - is interpreted as revealing a like or dislike. Yet, it could simply reflect the higher salience of Obama in today's media (see ref. ${ }^{65}$ for more details on salience). Information about prior exposure to cultural repertoires is required to determine whether a shorter association time is due to the lack of exposure to the designated item or the actual reflection of a bias against it.

Documenting predominant schemas can help IAT scholars to disentangle results due to salience and those resulting from evaluation. Cognitive psychologists could incorporate data about attitudes towards, and prior exposure to, specific groups based on the network-based questions found in surveys such as the General Social Survey to assess salience due to prior exposure that comes from network diversity. Historical and qualitative studies of changes in the stigmatization of groups could also inform the interpretations of IAT results: studies documenting the stigmatization of obesity ${ }^{66}$ can be used to point scholars to relevant questions about the respondent's prior awareness of and disposition towards a particular group. Interdisciplinary collaboration could further improve research by building on the work of scholars who examine how socially constructed logics prompt internal cognitive processes: for instance, one could refer to the work done by Stephens and colleagues ${ }^{15}$, who examined how "an intervention might teach students about the assumptions embedded in the university culture" (page 628, ref. ${ }^{15}$ ). While cultural sociologists can better understand how culture works by considering automatic cognition in their own analyses ${ }^{67}$, we propose that collaboration would also benefit IAT researchers, providing opportunities to refine their findings and the interpretations of their results. By taking into account cultural schemas in a more systematic manner, researchers using the IAT can open the black box of evaluative procedure to the benefit of psychologists and sociologists alike.

A more systematic inclusion of cultural repertoires in IAT studies could also have important effects for the practitioners who have been using the test to promote workplace inclusion. Addressing discrimination within organizations may require transforming durable institutional scripts rather than relying on the repeated administration of IATs in diversity training, which has been shown to have limited effect or unintended negative results ${ }^{68}$.

\section{Discussion and future agenda}

Engagement with the cultural repertoires that structure human judgement and behaviours would enrich the research areas we have discussed here. Each programme assumes an ahistorical or 'natural' set of cultural referents, which is contradicted by research that demonstrates cultural variation shaped by complex sociocultural pathways. While space limitations prevent a full review 
of relevant pathways, our discussion has focused on how cultural repertoires influence evaluation and decision-making. To develop more complete causal models and accurately interpret results, it is imperative that we build stronger bridges between cognitive psychology and studies of cultural repertoires.

What are the consequences of neglecting cultural variation for the tasks these fields define for themselves? Without considering how the poor prioritize multiple scarce resources, the $\mathrm{CB}$ model cannot offer causal explanations for decisions, given the many overlapping scarce resources defining poverty. Dual-process moral cognition aims to understand the universal structures of moral judgement but relies on culturally specific moral schemas, such that its predictions for moral decision-making apply only to individuals whose cultural repertoires match those baked into the model. Without investigating varying degrees to which groups are stigmatized, IAT scholars cannot fully interpret their results and distinguish between variations resulting from salience and evaluation. Interdisciplinary collaboration could yield a more accurate understanding of the cognitive dimensions of poverty, ethics and discrimination.

The social sciences have been moving away from a radical opposition between emic studies of culture 'in the wild' and lab experiments. Following DiMaggio ${ }^{69}$, Zerubavel $^{70}$ and others, cultural sociologists are building a bridge towards cognitive science by incorporating the DPM of cognition ${ }^{71}$, embodiment ${ }^{72}$, networks ${ }^{73}$, implicit bias ${ }^{74}$ and emotions into cultural sociology. Shepherd ${ }^{67}$ in particular has considered the impact of symbols and media, place, situation and networks in IAT research. It is crucial that we continue to consider how cognition both responds to and helps to produce the scripts and schemas available in a particular cultural environment. But creating a bridge requires building from both ends. We believe that cognitive psychologists can also contribute.

The most fruitful insights will come from interdisciplinary dialogue at the intersection of group-level repertoires and universal cognitive processes. In the study of poverty, cultural sociologists can build on existing cognitive research to investigate the scripts, schemas and frames that may lead poor people in specific contexts to prioritize scarce resources in diverse ways (for example, refs ${ }^{23,30,75}$ ). Cognitive psychologists can improve research designs with broader questions about the meaning and valuation of scarce resources (for example, ref. $\left.{ }^{76}\right)$. DPM scientists should incorporate findings from the sociology, anthropology and cultural psychology of morality to study how repertoires structure moral intuitions or reflection. IAT researchers could better interpret test results by incorporating fine-grained questions about exposure and salience in their research design.

This Perspective also serves as a warning concerning the limitations of three lines of cognitive research particularly popular among policymakers. Cognitive psychology can enrich our understanding of the effects of poverty and the role of individual decisions in reproducing inequality. However, poverty cannot be addressed by urging the poor to engage in deliberative thinking ${ }^{77}$ : reducing poverty requires that public policies enhance material redistribution and social recognition. Promoting ethical decision-making and resolving moral conflicts will require changes in repertoires about morality, rather than shifting modes of cognition ${ }^{78}$. Finally, we are more likely to address discrimination by gradually changing cultural narratives that stigmatize particular groups (for example, Clair, Daniel and Lamont ${ }^{66}$ on the declining stigmatization of people with HIV/AIDS) than by simply sensitizing individuals to their own subconscious biases.

Cognition plays an important role in social life. Putting cognition within a broader cultural context can help us move beyond the appealing simplicity of individual models and address critical social problems of poverty, morality and discrimination by engaging the structures that unevenly distribute material and cultural resources.
Received: 27 April 2017; Accepted: 12 October 2017; Published online: 20 November 2017

\section{References}

1. Perrin, A. J. \& Lee, H. The undertheorized environment: sociological theory and the ontology of behavioral genetics. Sociol. Perspect. 50, 303-322 (2007).

2. Abend, G. The Moral Background: An Inquiry into the History of Business Ethics (Princeton Univ. Press, Princeton, NJ, 2014).

3. Bruch, E. \& Feinberg, F. Decision-making processes in social contexts. Annu. Rev. Sociol. 43, 207-227 (2017).

4. Lamont, M. \& Small, M. L. in The Colors of Poverty: Why Racial and Ethnic Disparities Persist (eds Lin, A. C. \& Harris, D. R.) 76-102 (Russell Sage Foundation, New York, NY, 2008)

5. Parsons, T. Societies: Evolutionary and Comparative Perspectives (Prentice-Hall, Englewood Cliffs, NJ, 1966).

6. Swidler, A. Culture in action: symbols and strategies. Am. Sociol. Rev. 51, 273-286 (1986)

7. Lamont, M. \& Thévenot, L. Rethinking Comparative Cultural Sociology: Repertoires of Evaluation in France and the United States (Cambridge Univ. Press, Cambridge, 2000).

8. Thornton, P. H., Ocasio, W. \& Lounsbury, M. The Institutional Logics Perspective: A New Approach to Culture, Structure, and Process (Oxford Univ. Press, Oxford, 2012).

9. Willis, P. E. Learning to Labor: How Working Class Kids Get Working Class Jobs (Columbia Univ. Press, New York, NY, 1977).

10. Hays, S. The Cultural Contradictions of Motherhood (Yale Univ. Press, New Haven, CT, 1998).

11. Buchtel, E. et al. Immorality East and West: are immoral behaviors especially harmful, or especially uncivilized? Pers. Soc. Psychol. Bull. 41 1382-1394 (2015).

12. Cole, M. Cultural Psychology: A Once and Future Discipline (Harvard Univ. Press, Cambridge, MA, 1996).

13. Markus, H. R. \& Kitayama, S. Culture and the self: implications for cognition, emotion, and motivation. Psychol. Rev. 98, 224-253 (1991).

14. Shweder, R. A., Much, N., Mahapatra, M. \& Park, L. in Morality and Health (eds Brandt, A. \& Rozin, P.) 119-172 (Routledge, London, 1997).

15. Stephens, N. M., Markus, H. R. \& Phillips, L. T. Social class culture cycles: how three gateway contexts shape selves and fuel inequality. Annu. Rev. Psychol. 65, 611-634 (2014).

16. Lamont, M. et al. Getting Respect: Responding to Stigma and Discrimination in the United States, Brazil, and Israel (Princeton Univ. Press, Princeton, NJ, 2016)

17. Lewis, O. Five Families: Mexican Case Studies in the Culture of Poverty (Basic Books, New York, NY, 1975).

18. Mullainathan, S. \& Shafir, E. Scarcity: Why Having Too Little Means So Much (Macmillan, New York, NY, 2013).

19. Bertrand, M., Mullainathan, S. \& Shafir, E. A behavioral-economics view of poverty. Am. Econ. Rev. 94, 419-423 (2004).

20. Mani, A., Mullainathan, S., Shafir, E. \& Zhao, J. Poverty impedes cognitive function. Science 341, 976-980 (2013).

21. Shah, A. K., Shafir, E. \& Mullainathan, S. Scarcity frames value. Psychol. Sci. 26, 402-412 (2015).

22. Desmond, M. Severe deprivation in America: an introduction. RSF 1 , 1-11 (2015).

23. Rao, V. Poverty and public celebrations in rural India. Ann. Am. Acad. Pol. Soc. Sci. 573, 85-104 (2001).

24. Desmond, M. Evicted: Poverty and Profit in the American City (Crown, New York, NY, 2016).

25. Waller, M. R. Viewing low-income fathers' ties to families through a cultural lens: insights for research and policy. Ann. Am. Acad. Pol. Soc. Sci. 629, 102-124 (2010).

26. Daminger, A., Hayes, J., Barrows, A. \& Wright, J. Poverty Interrupted: Applying Behavioral Science to the Context of Chronic Scarcity (ideas42, New York, NY, 2015).

27. Rosen, E. Horizontal immobility: how narratives of neighborhood violence shape housing decisions. Am. Sociol. Rev. 82, 270-296 (2017).

28. Blair-Loy, M. Cultural constructions of family schemas: the case of women finance executives. Gend. Soc. 15, 687-709 (2001).

29. Bandelj, N., Wherry, F. F. \& Zelizer, V. A. Money Talks: Explaining How Money Really Works (Princeton Univ. Press, Princeton, NJ, 2017).

30. Daniel, C. Economic constraints on taste formation and the true cost of healthy eating. Soc. Sci. Med. 148, 34-41 (2016).

31. Damasio, A. R. Descartes' Error: Emotion, Rationality and the Human Brain (Norton, Boston, MA, 1994).

32. Greene, J. \& Haidt, J. How (and where) does moral judgment work? Trends Cogn. Sci. 6, 517-523 (2002).

33. Haidt, J. The emotional dog and its rational tail: a social intuitionist approach to moral judgment. Psychol. Rev. 108, 814-834 (2001). 
34. Cushman, F. Action, outcome, and value: a dual-system framework for morality. Pers. Soc. Psychol. Rev. 17, 273-292 (2013).

35. Greene, J. D. Why are VMPFC patients more utilitarian? A dual-process theory of moral judgment explains. Trends Cogn. Sci. 11, 322-323 (2007).

36. Greene, J. Moral Tribes: Emotion, Reason and the Gap between Us and Them (Penguin Press, New York, NY, 2013).

37. Bartels, D. M. \& Pizarro, D. A. The mismeasure of morals: antisocial personality traits predict utilitarian responses to moral dilemmas. Cognition 121, 154-161 (2011)

38. Conway, P. \& Gawronski, B. Deontological and utilitarian inclinations in moral decision making: a process dissociation approach. J. Pers. Soc. Psychol. 104, 216-235 (2013)

39. Cushman, F., Young, L. \& Hauser, M. The role of conscious reasoning and intuition in moral judgment: testing three principles of harm. Psychol. Sci. 17, 1082-1089 (2006).

40. Greene, J. D., Morelli, S. A., Lowenberg, K., Nystrom, L. E. \& Cohen, J. D. Cognitive load selectively interferes with utilitarian moral judgment. Cognition 107, 1144-1154 (2008).

41. Greene, J. D. et al. Pushing moral buttons: the interaction between personal force and intention in moral judgment. Cognition 111, 364-371 (2009).

42. Hauser, M. D. Moral Minds: How Nature Designed Our Universal Sense of Right and Wrong (HarperCollins Publishers, New York, NY, 2006).

43. Xiang, X. Would the Buddha Push the Man off the Footbridge? Systematic Variations in the Moral Judgment and Punishment Tendencies of Han Chinese, Tibetans and Americans (Harvard University, Cambridge, MA, 2014).

44. Cushman, F., Young, L. \& Greene, J. D. in The Moral Psychology Handbook (ed. Doris, J. M.) 47-71 (Oxford Univ. Press, Oxford, 2010)

45. Henrich, J. et al. (eds) Foundations of Human Sociality: Economic Experiments and Ethnographic Evidence from Fifteen Small-Scale Societies (Oxford Univ. Press, Oxford, 2004)

46. Flanagan, O. The Geography of Morals: Varieties of Moral Possibility (Oxford Univ. Press, Oxford, 2016)

47. Lamont, M. Money, Morals, and Manners: The Culture of the French and American Upper-Middle Class (Univ. Chicago Press, Chicago, IL, 1992).

48. Lamont, M. The Dignity of Working Men: Morality and the Boundaries of Race, Class, and Immigration (Russell Sage Foundation, New York, NY \& Harvard Univ. Press, Cambridge, MA, 2000).

49. Boltanski, L. \& Thévenot, L. On Justification: Economies of Worth (Princeton Univ. Press, Princeton, NJ, 2006).

50. McAdams, D. P. et al. Family metaphors and moral intuitions: how conservative and liberals narrate their lives. J. Pers. Soc. Psychol. 95, 978-990 (2008).

51. Keane, W. Ethical Life: Its Natural and Social Histories (Princeton Univ. Press, Princeton, NJ, 2015)

52. Haidt, J., Bjorklund, F. \& Murphy, S. Moral Dumbfounding: When Intuition Finds No Reason (Univ. Virginia, Charlottesville, VA, 2000); http://faculty. virginia.edu/haidtlab/articles/manuscripts/haidt.bjorklund.working-paper. when intuition finds no reason.pub603.doc.

53. Lowes, S., Nunn, N., Robinson, J. A. \& Weigel, J. Understanding ethnic identity in Africa: evidence from the implicit association test (IAT). Am. Econ. Rev. 105, 340-345 (2015).

54. Nosek, B. A., Bar-Anan, Y., Sriram, N., Axt, J. \& Greenwald, A. G. Understanding and using the brief implicit association test: recommended scoring procedures. PLOS ONE 9, el10938 (2014).

55. Greenwald, A. G., McGhee, D. E. \& Schwartz, J. L. K. Measuring individual differences in implicit cognition: the implicit association test. J. Pers. Soc. Psychol. 74, 1464-1480 (1998).

56. Oswald, F. L., Mitchell, G., Blanton, H., Jaccard, J. \& Tetlock, P. E. Using the IAT to predict ethnic and racial discrimination: small effect sizes of unknown societal significance. J. Pers. Soc. Psychol. 108, 562-571 (2015).

57. Blanton, H., Jaccard, J., Christie, C. \& Gonzales, P. M. Plausible assumptions, questionable assumptions and post hoc rationalizations: will the real IAT, please stand up? J. Exp. Soc. Psychol. 43, 399-409 (2007).

58. Kaufman, S. B. Does the implicit association test (IAT) really measure racial prejudice? Probably not. Psychology Today (28 January 2011); http://www. psychologytoday.com/blog/beautiful-minds/201101/does-the-implicitassociation-test-iat-really-measure-racial-prejudice.
59. Greenwald, A. G., Banaji, M. R. \& Nosek, B. A. Statistically small effects of the implicit association test can have societally large effects. J. Pers. Soc. Psychol. 108, 553-561 (2015).

60. McConnell, A. R. \& Leibold, J. M. Relations among the implicit association test, discriminatory behavior, and explicit measures of racial attitudes. J. Exp. Soc. Psychol. 37, 435-442 (2001).

61. Ziegert, J. C. \& Hanges, P. J. Employment discrimination: the role of implicit attitudes, motivation, and a climate for racial bias. J. Appl. Psychol. 90, 553-562 (2005)

62. Bargh, J. A. \& Chartrand, T. L. The unbearable automaticity of being. Am. Psychol. 54, 462-479 (1999).

63. Dunham, Y., Chen, E. E. \& Banaji, M. R. Two signatures of implicit intergroup attitudes: developmental invariance and early enculturation. Psychol. Sci. 24, 860-868 (2013).

64. Banaji, M. R. \& Greenwald, A. G. Blindspot: Hidden Biases of Good People (Delacorte Press, New York, NY, 2013).

65. Matthews, S. A. The salience of neighborhood: some lessons from sociology. Am. J. Prev. Med. 34, 257-259 (2008).

66. Clair, M., Daniel, C. \& Lamont, M. Destigmatization and health: cultural constructions and the long-term reduction of stigma. Soc. Sci. Med. 165, 223-232 (2016)

67. Shepherd, $H$. The cultural context of cognition: what the implicit association test tells us about how culture works. Sociol. Forum $\mathbf{2 6}$ 121-143 (2011).

68. Dobbin, F. \& Kalev, A. Why diversity programs fail. Harv. Bus. Rev. 94, 52-60 (2016).

69. DiMaggio, P. Culture and cognition. Annu. Rev. Sociol. 23, 263-287 (1997).

70. Zerubavel, E. Social Mindscapes: An Invitation to Cognitive Sociology (Harvard Univ. Press, Cambridge, MA, 1997).

71. Vaisey, S. Motivation and justification: a dual-process model of culture in action. Am. J. Sociol. 114, 1675-1715 (2009).

72. Martin, J. L. Social Structures (Princeton Univ. Press, Princeton, NJ, 2009).

73. Mohr, J. W. Measuring meaning structures. Annu. Rev. Sociol. 24, 345-370 (1998)

74. Warikoo, N., Sinclair, S., Fei, J. \& Jacoby-Senghor, D. Examining racial bias in education: a new approach. Educ. Res. 45, 508-514 (2016).

75. Sanyal, P. Credit to Capabilities: A Sociological Study of Microcredit Groups in India (Cambridge Univ. Press, Cambridge, 2014).

76. Srivastava, S. B. \& Banaji, M. R. Culture, cognition, and collaborative networks in organizations. Am. Sociol. Rev. 76, 207-233 (2011).

77. Babcock, E. D. Using Brain Science to Design New Pathways out of Poverty (Crittenton Women's Union, Boston, MA, 2014).

78. Lamont, M. Addressing the Recognition Gap: Destigmatization and the Reduction of Inequality (Presidential Address, 112th Annual Meeting of the American Sociological Association, Toronto, ON, 2017); https://vimeo. com/230762647.

79. Cushman, F. \& Greene, J. D. Finding faults: how moral dilemmas illuminate cognitive structure. Soc. Neurosci. 7, 269-279 (2012).

\section{Acknowledgements}

We thank G. Abend, B. Bonikowski, K. Cerulo, C. Daniel, P. DiMaggio, F. Dobbin H. Gardner, P. Hall, H. Haste, S. Lukes, J. Mijs, A. Perrin and A. Wilson for their helpful feedback on an earlier version of the paper. M.L. acknowledges support from the Canadian Institute for Advanced Research.

\section{Competing interests}

The authors declare no competing interests.

\section{Additional information}

Reprints and permissions information is available at www.nature.com/reprints.

Correspondence and requests for materials should be addressed to M.L.

Publisher's note: Springer Nature remains neutral with regard to jurisdictional claims in published maps and institutional affiliations. 